Pacific Journal of Mathematics

ELEMENTARY SOLUTIONS OF DIFFERENTIAL EQUATIONS 


\title{
ELEMENTARY SOLUTIONS OF DIFFERENTIAL EQUATIONS
}

\author{
Michael F. Singer
}

\begin{abstract}
In this paper we deal with the problem: when does a differential equation have an elementary solution, that is a solution which can be expressed in terms of algebraic operations, logarithms and exponentials? As an application of our theorem, we give necessary and sufficient conditions for a certain class of first order differential equations to have elementary solutions.
\end{abstract}

For the simplest differential equation $y^{\prime}=\alpha$, where $\alpha$ is an algebraic function, Liouville showed that if such an equation has an elementary solution, then this solution is an algebraic function plus a sum of constant multiples of logarithms of algebraic functions. In his paper, "Liouville's Theorem on Functions with Elementary Integrals", Pacific J. of Math., 24 No. 1, Rosenlicht showed how this theorem can be handled algebraically and generalized. We will use Rosenlicht's methods to show that if an arbitrary algebraic differential equation has an elementary solution, this solution must be of a special form.

An (ordinary) differential field is a field $K$ and a map ' $: K \rightarrow K$ called a derivation, which satisfies $(a+b)^{\prime}=a^{\prime}+b^{\prime}$ and $(a b)^{\prime}=$ $a^{\prime} b+a b^{\prime}$ for all $a, b$ in $K$. For example, a field of functions, meromorphic in some region of the plane, with the usual differentiation, is such a field. A differential subfield $k$ of $K$ is a subfield which is closed under the derivation. If $c$ is in $K$ and $c^{\prime}=0$ then $c$ is called a constant of $K$. The set of constants can be seen to form a subfield of $K$. In this paper all fields will be of characteristic 0. By a differential equation of order $n$ over $k$, we mean an expression of the form $f\left(y, y^{\prime}, \cdots, y^{(n)}\right)=0$ where $f$ is a polynomial, with coefficients in $k$, in the variables $y, y^{\prime}, \cdots, y^{(n)}$ with $y^{(n)}$ actually appearing. An element $u$ of $K$ is said to satisfy such an equation if $f\left(u, u^{\prime}, \cdots\right.$, $\left.u^{(n)}\right)=0$ where $u^{(i)}$ is the $i$ th derivative of $u$. We note that if $u$ satisfies a differential equation of order $n$, then the field $k\left(u, u^{\prime}, \cdots, u^{(n)}\right)$ is a differential field of transcendence degree at most $n$. To see that it is closed under the derivation, note that by differentiating the equation $f\left(u, u^{\prime}, \cdots, u^{(n)}\right)=0$ we can solve for $u^{(n+1)}$ in terms of lower derivatives of $u$.

If $k \subset K$ are differential fields an element $u(u \neq 0)$ in $K$ is called an elementary integral (exponential of an elementary integral) with respect to $k$ if there exist elements $v_{0}, v_{1}, \cdots, v_{n}$ in $k$ and $c_{1}, c_{2}, \cdots, c_{n}$ 
constants of $k$ such that

$$
\begin{gathered}
u^{\prime}=v_{0}^{\prime}+\sum_{i=1}^{n} c_{i} \frac{v_{i}^{\prime}}{v_{i}} \\
\left(\frac{u^{\prime}}{u}=v_{0}^{\prime}+\sum_{i=1}^{n} c_{i} \frac{v_{i}^{\prime}}{v_{i}}\right) .
\end{gathered}
$$

For elements $u, v$ in $K$ with $u \neq 0$, we say $u$ is an exponential of $v$ or equivalently $v$ is a logarithm of $u$ if $v^{\prime}=u^{\prime}{ }_{1}^{\prime} u$. Note that if $u$ is in $k$ ( $v$ is in $k$ ) then $v$ is an elementary integral ( $u$ is an exponential of an elementary integral) with respect to $k$. $K$ is called a generalized elementary extension of $k$ if

(1) $K$ and $k$ have the same field of constants, and

(2) there exists a tower of differential fields $k=K_{0} \subset K_{1} \subset \cdots \subset$ $K_{n}=K$ where $K_{i}=K_{i-1}\left(u_{i}\right)$ and $u_{i}$ is either algebraic over $K_{\imath-1}$ or an elementary integral with respect to $K_{i-1}$ or an exponential of an elementary integral with respect to $K_{i-1}$.

If $K$ is a generalized elementary extension of $k$ and satisfies the additional property that each of the above $u_{i}$ is either algebraic over $K_{i-1}$ or a logarithm or exponential of an element in $K_{i-1}$, we say $K$ is an elementary extension of $k$. It was shown in [8] that every generalized elementary extension of $k$ lies in an elementary extension of $k$. Intuitively, one just has to add enough logarithms into a generalized elementary extension $K$, making sure not to extend the constants, to get an elementary extension containing $K$. We say an element $w$ of some differential extension of $k$ is elementary with respect to $k$ if it lies in some elementary extension of $k$.

Generalized elementary extensions were introduced to deal with the following phenomenon: Liouville's theorem [5], says that if $u$ is elementary with respect to $k$ and $u^{\prime}$ is in $k$, then

$$
u^{\prime}=v_{0}^{\prime}+\sum_{i=1}^{n} c_{i} \frac{v_{i}^{\prime}}{v_{i}}
$$

for some elements $v_{i}$ in $k$ and constants $c_{i}$. Another way of saying this is: if an integral $u$ of an element of $k$ is elementary, then $u$ is an elementary integral, (this, of course, was why elementary integrals were so named). In general we have no bound on the transcendence degree of the smallest elementary extension of $k$ containing $u$, because we don't know how many logarithms (all possibly algebraically independent) we need to adjoint to $k$ to insure that we get $u$. Yet, we know that $u$ lies in a generalized elementary extension of transcendence degree at most one over $k$. The main theorem of this paper 
says that this is true in general; if a differential equation over $k$ of order $n$ has a solution in a generalized elementary extension of $k$, this solution lies in a generalized elementary extension of transcendence degree less than or equal to $n$ over $k$.

Before I proceed, I need a technical fact about generalized elementary extensions. We say an element $u$ in $K$ is a good elementary integral with respect to $k \subset K$ if

$$
u^{\prime}=v_{0}^{\prime}+\sum_{i=1}^{n} c_{i} \frac{v_{i}^{\prime}}{v_{i}}
$$

with $v_{i}$ in $k$ and $\left\{c_{1}, c_{2}, \cdots, c_{n}\right\}$ a $Q$-linearly independent set of constants. An element $u \neq 0$ in $K$ is said to be a good exponential of an elementary integral with respect to $k \subset K$ if

$$
\frac{u^{\prime}}{u}=v_{0}^{\prime}+\sum_{i=1}^{n} c_{i} \frac{v_{i}^{\prime}}{v_{i}}
$$

with the $v_{i}$ in $k$ and $\left\{1, c_{1}, c_{2}, \cdots, c_{n}\right\}$ a $Q$-linearly independent set of constants. A generalized elementary extension $K$ of $k$ is said to be good if the elementary integrals and exponentials of elementary integrals used in building up the tower to $K$ are good. I claim that for any generalized elementary extension $K$ of $k$ there is a tower of fields $k=K_{0}^{*} \subset K_{1}^{*} \subset \cdots \subset K_{n}^{*}=K$ which turns $K$ into a good elementary extension of $k$. It is enough to show that for $E=F(\theta)$, where $\theta$ is an elementary integral or exponential of an elementary integral, we can make $E$ into a good elementary extension of $F$. Let

$$
\theta^{\prime}=v_{0}^{\prime}+\sum_{i=1}^{k} c_{i} \frac{v_{i}^{\prime}}{v_{i}}
$$

and assume that $c_{i}$ 's are linearly dependent over $Q$. We can assume that $c_{k}$ depends linearly on $c_{1}, \cdots, c_{k-1}$ and let

$$
c_{k}=\frac{\left(n_{1} c_{1}+\cdots+n_{k-1} c_{k-1}\right)}{n}
$$

with $n, n_{1}, \cdots, n_{k-1}$ integers, then

$$
\theta^{\prime}=v_{0}^{\prime}+\sum_{i=1}^{k-1} \frac{c_{i}}{n} \frac{\left(v_{2}^{n} v_{k}^{n_{i}}\right)^{\prime}}{v_{i}^{n} v_{k}^{n_{i}}}
$$

Continuing in this way we can eventually arrive at an expression

$$
\theta^{\prime}=v_{0}^{\prime}+\sum_{i=1}^{j} d_{i} \frac{u_{i}^{\prime}}{u_{i}}
$$


where $\left\{d_{1}, \cdots, d_{j}\right\}$ is a $Q$-linearly independent set of constants and $u_{0}, u_{1}, \cdots, u_{j}$ are elements of $k$. So using this expression we see that $F(\theta)$ is a good elementary extension of $F$. Now assume that $\theta$ satisfies

$$
\frac{\theta^{\prime}}{\theta}=v_{0}^{\prime}+\sum_{i=1}^{k} c_{i} \frac{v_{i}^{\prime}}{v_{i}}
$$

and suppose $\left\{1, c_{1}, \cdots, c_{k}\right\}$ are $Q$-linearly dependent. As before assume that $c_{k}$ depends linearly on $1, c_{1}, \cdots, c_{k-1}$, and let, $c_{k}=\left(n_{0}+n_{1} c_{1}+\right.$ $\left.\cdots+n_{k-1} c_{k-1}\right) / n$ where $n, n_{0}, n_{1}, \cdots, n_{k-1}$ are integers, We can then write

$$
\frac{\left(\theta^{n} v_{k}^{-n_{0}}\right)^{\prime}}{\theta^{n} v_{k}^{-n_{0}}}=n v_{0}^{\prime}+\sum_{i=1}^{k-1} c_{i} \frac{\left(v_{i}^{n} v_{k}^{n_{i}}\right)}{v_{i}^{n} v_{k}^{n}}
$$

Letting $\theta^{*}=\theta^{n} v_{k}^{-n_{0}}, u_{i}=v_{i}^{n} v_{k}^{n_{i}}, u_{0}=n v_{0}$, we get

$$
\frac{\theta^{* \prime}}{\theta^{*}}=u_{0}^{\prime}+\sum_{i=1}^{k-1} c_{i} \frac{u_{i}^{\prime}}{u_{i}}
$$

Note that $\theta$ is algebraic over $F\left(\theta^{*}\right)$. Continuing in this way we eventually get to the stage where $\left\{1, c_{1}, \cdots, c_{j}\right\}$ are linearly independent over $Q$, and $\theta$ algebraic over $F\left(\theta^{*}\right)$ so $F \subset F\left(\theta^{*}\right) \subset F(\theta)$ is a good generalized elementary extension of $F$. In conclusion, we have shown that if $\theta$ is an elementary integral with respect to $F$, then it is a good elementary integral with respect to $F$, and that if $\theta$ is an exponential of an elementary integral with respect to $F$ then some $a \theta^{n}\left(n \in Z, n \neq 0, a \in F^{*}\right)$ is a good exponential of an elementary integral with respect to $F$. This allows us to exhibit any elementary extension $K$ of $k$ as a good elementary extension of $k$.

The proof of the theorem relies on a fact about algebraic dependence of certain elements in generalized elementary extension (the lemma below). This in turn relies on the following proposition, whose proof can be found in [6].

Proposition. Let $L$ be a differential field and $K$ a differential extension field with the same constants as $L$, which is furthermore algebraic over $k(\zeta)$ for some $\zeta$ in $K$. Suppose that $c_{1}, \cdots, c_{n}$ are constants which are linearly independent over $Q$ and $u_{1}, \cdots, u_{n}, v$ in $K\left(u_{i} \neq 0\right.$ for all $\left.i\right)$. Suppose further that

$$
v^{\prime}-\sum_{i=1}^{n} c_{i} \frac{u_{i}^{\prime}}{u_{i}}
$$

is in $L$. If $\zeta^{\prime}$ is in $L$, then $u_{1}, \cdots, u_{n}$ are in $\bar{L}$ (the algebraic closure of $L$ in $K$ ) and $v=c \zeta+d$, where $c$ is a constant and $d$ is in $\bar{L}$. 
If $\zeta^{\prime} / \zeta$ is in $L$, then we have $v$ in $\bar{L}$ and there are integers $\nu_{0}, \nu_{1}$, $\cdots, \nu_{n}$ with $\nu_{0} \neq 0$ such that $u_{i}^{\nu_{0}^{\prime} \zeta^{\nu_{i}}}$ is in $\bar{L}$ for $1 \leqq i \leqq n$.

Lemma. Let $k \subset L \subset K$ be differential fields with the same constants. Let $\zeta, \eta$ be elements of $K$, algebraically dependent over $L$ such that $\zeta$ is an elementary integral or an exponential of an elementary integral with respect to $\bar{k}$, the algebraic closure of $k$ in $K$ and $\eta$ is a good elementary integral or a good exponential of an elementary integral with respect to $\overline{k(\zeta)}$. We can then find a $\xi$ in $K$ such that

(1) $\xi$ is an elementary integral or an exponential of an elementary integral with respect to $\bar{k}$.

(2) $\zeta$ and $\eta$ are algebraically dependent over $k(\xi)$.

(3) $\xi$ is algebraic over $L$.

Furthermore, if $\zeta, \eta$ are both elementary integrals, so is $\xi$ and if $\zeta, \eta$ are both exponentials of elementary integrals then so is $\xi$.

Proof. First note that we can assume that $\zeta$ is not algebraic over $L$, otherwise we could take $\zeta$ for our $\xi$. From this we can conclude that $\overline{k(\zeta)} \cap \bar{L}=\bar{k}$. The proof now proceeds by considering the following four cases:

Case 1. Assume

$$
\zeta^{\prime}=\Sigma a_{i} \frac{s_{i}^{\prime}}{s_{i}}+t^{\prime} \quad \text { and } \quad \eta^{\prime}=\Sigma b_{i} \frac{u_{i}^{\prime}}{u_{i}}+v^{\prime}
$$

where $s_{i}, t$ are in $\bar{k}$ and $u_{i}, v$ are in $\overline{k(\zeta)}$ and $\left\{b_{i}\right\}$ is a $Q$-linearly independent set of constants. We can apply the proposition to the expression

$$
\Sigma b_{i} \frac{u_{i}^{\prime}}{u_{i}}+(v-\eta)^{\prime}=0
$$

with respect to the fields $L \subset L(\zeta) \subset K$ and conclude that $u_{1}, \cdots, u_{n}$ are in $\bar{L}$ and $v-\eta=c \zeta+d$ where $c$ is a constant and $d$ is in $\bar{L}$. Let $\xi=d=v-\eta-c \zeta$. Conditions (2) and (3) are then clearly satisfied. Note that since $u_{1}, \cdots, u_{n}$ are in $\overline{k(\zeta)} \cap \bar{L}$, they are in $\bar{k}$. We have

$$
\xi^{\prime}=v^{\prime}-\eta^{\prime}-c \zeta^{\prime}=-\Sigma b_{i} \frac{u_{i}^{\prime}}{u_{i}}-c \Sigma a_{i} \frac{s_{i}^{\prime}}{s_{i}}-c t^{\prime}
$$

Since $u_{i}, s_{i}, t$ are in $\bar{k}, \xi$ is an elementary integral with respect to $\bar{k}$, so we have (1). 
Case 2. Assume that

$$
\zeta^{\prime}=\Sigma a_{i} \frac{s_{i}^{\prime}}{s_{i}}+t^{\prime} \text { and } \frac{\eta^{\prime}}{\eta}=\Sigma b_{i} \frac{u_{i}^{\prime}}{u_{i}}+v^{\prime}
$$

where $s_{i}, t, u_{i}, v$ are as before and $\left\{1, b_{i}\right\}$ is a $Q$-linearly independent set of constants. We can apply the proposition to the expression

$$
-\frac{\eta^{\prime}}{\eta}+\Sigma b_{i} \frac{u_{i}^{\prime}}{u_{i}}+v^{\prime}=0
$$

and conclude that the $u_{i}$ and $\eta$ are in $\bar{L}$ and $v=c \zeta+d$ with $d$ in $\bar{L}$ and $c$ a constant. Let $\xi=\eta$. Conditions (2) and (3) are then satisfied. Since the $s_{i}$ and $d=V-c \zeta$ are in $\bar{L} \cap \overline{k(\zeta)}=\bar{k}$ and

$$
\begin{aligned}
\frac{\xi^{\prime}}{\xi} & =\frac{\eta^{\prime}}{\eta}=\Sigma b_{i} \frac{u_{i}^{\prime}}{u_{i}}+v^{\prime}=\Sigma b_{i} \frac{u_{i}^{\prime}}{u_{i}}+c \zeta^{\prime}+d^{\prime} \\
& =\Sigma b_{i} \frac{u_{i}^{\prime}}{u_{i}}+c\left(\Sigma a_{i} \frac{s_{i}^{\prime}}{s_{i}}+t^{\prime}\right)+d^{\prime}
\end{aligned}
$$

we have that $\xi$ is an exponential of an elementary integral over $\bar{k}$.

Case 3. Assume

$$
\frac{\zeta^{\prime}}{\zeta}=\Sigma a_{i} \frac{s_{i}^{\prime}}{s_{i}}+t^{\prime} \text { and } \eta^{\prime}=\Sigma b_{i} \frac{u_{i}^{\prime}}{u_{i}}+v^{\prime}
$$

with $u_{i}, v, s_{i}, t$ as before and $\left\{b_{i}\right\}$ a $Q$-linearly independent set of constants. We can apply the proposition to the expression

$$
\Sigma b_{i} \frac{u_{i}^{\prime}}{u_{i}}+(v-\eta)^{\prime}=0
$$

and conclude that $v-\eta$ is in $\bar{L}$ and that there are integers $\nu_{0}, \cdots, \nu_{n}$ with $\nu_{0} \neq 0$ such that $u_{i}^{\nu_{0} \zeta^{\nu_{i}}}$ is in $\bar{L}$. Let $\xi=\nu_{0}(\eta-v)$. $\xi$ then satisfies (2) and (3). Each $u_{i}^{\nu} \zeta^{\nu_{i}}$ is in $\overline{k(\zeta)} \cap \bar{L}=\bar{k}$ and

$$
\xi^{\prime}=\nu_{0}\left(\eta^{\prime}-v^{\prime}\right)=\Sigma b_{i} \frac{\left(u_{i}^{\nu_{0}} \zeta^{\nu_{2}}\right)^{\prime}}{u_{i}^{\nu_{0} \zeta^{\nu_{i}}}}-\left(\sum \nu_{i} b_{i}\right)\left(\sum a_{i} \frac{s_{i}^{\prime}}{s_{i}}+t^{\prime}\right)
$$

so $\xi$ is an elementary integral with respect to $\bar{k}$.

Case 4. Assume

$$
\frac{\zeta^{\prime}}{\zeta}=\Sigma a_{i} \frac{s_{i}^{\prime}}{s_{i}}+t^{\prime} \text { and } \frac{\eta^{\prime}}{\eta}=\Sigma b_{i} \frac{u_{i}^{\prime}}{u_{i}}+v^{\prime}
$$

with $u_{i}, v, s_{i}, t$ as before and $\left\{1, b_{i}\right\}$ a $Q$-linearly independent set of constants. We can apply the proposition to the expression 


$$
-\frac{\eta^{\prime}}{\eta}+\Sigma b_{\imath} \frac{u_{i}^{\prime}}{u_{i}}+v^{\prime}=0
$$

and conclude that $v$ is in $\bar{L}$ and that there are integers $\nu_{0}, \nu_{1}, \cdots$, $\nu_{n+1}$ with $\nu_{0} \neq 0$ such that the $u_{i}^{\nu} \zeta^{\nu_{i}}$ and $\eta^{\nu_{0}} \zeta^{\nu_{n}+1}$ are in $\bar{L}$. Let $\xi=\eta^{\nu} \zeta^{\nu} n+1$. (2) and (3) are then satisfied

$$
\begin{aligned}
& \frac{\xi^{\prime}}{\xi}=\frac{\left(\eta^{\nu}\left(\zeta^{\nu} n+1\right)^{\prime}\right.}{\eta^{\nu} \zeta^{\nu} n+1}=\Sigma b_{i} \frac{\left(u_{i}^{\nu} \zeta^{\nu} \zeta^{\nu}\right)^{\prime}}{u_{\imath}^{\nu_{11} \zeta^{\nu_{i}}}}+\nu_{0} v^{\prime} \\
& +\left(\nu_{n+1}-\Sigma b_{i} \nu_{i}\right)\left(\Sigma a_{\imath} \frac{s_{i}^{\prime}}{s_{i}}+t^{\prime}\right)
\end{aligned}
$$

so $\xi$ is an exponential of an elementary integral.

THEOREM. Let $E$ be a differential field of transcendence degree $n$ over a differential field $F$. If $E$ lies in a generalized elementary extension $K$ of $F$, then $E$ lies in a generalized elementary extension of $F$ of transcendence degree $n$ over $F$. Furthermore, if $K=$ $F\left(\theta_{1}, \cdots, \theta_{N}\right)$ where each $\theta_{i}$ is algebraic or an elementary integral with respect to $F\left(\theta_{1}, \cdots, \theta_{i-1}\right)$, then $E$ lies in a generalized elementary extension of $F$ of transcendence degree $n$ which is likewise generated only by elements which are algebraic or elementary integrals. A similar statement holds if we restrict each $\theta_{i}$ to be algebraic or an exponential of an elementary integral with respect to $F\left(\theta_{1}, \cdots, \theta_{i-1}\right)$.

Proof. If $n=0$, then the theorem is a triviality. Therefore, we can assume that the transcendence degree of $E$ over $F$ is $\geqq 1$. If $F$ consisted only of constants, then any generalized elementary extension would coincide with $F$. So we can assume $F$ contains a nonconstant. Furthermore, by the primitive element theorem for differential fields [7], we can conclude that $E=F\left(y, y^{\prime}, \cdots, y^{(n)}\right)$ for some element $y$ of $E$. Note that $y, y^{\prime}, \cdots, y^{(n-1)}$ forms a transcendence base for $E$ over $F$. Now the proof proceeds by induction on $n$. Although the proof could be written to suppress the $n=1$ step, we include it here in the hope that it will aid in understanding the induction step.

$n=1$. Let $E=F\left(y, y^{\prime}\right)$ with $y^{\prime}$ algebraic over $F(y)$. Let $K$ be a generalized elementary extension of $F$, containing $E$, whose transcendence degree over $F$ is minimal with respect to all such extensions. Using the facts about good elementary extension developed in the paragraphs preceding the Proposition, we can pick a transcendence basis $\theta_{1}, \cdots, \theta_{m}$ such that each $\theta_{i}$ is a good elementary integral or a good exponential of an elementary integral with respect to some algebraic extension of $F\left(\theta_{1}, \cdots, \theta_{i-1}\right)$. Now assume $m>1$ 
and we will work towards a contradiction. We will apply the lemma, so let $k$ be the algebraic closure of $F\left(\theta_{1}, \cdots, \theta_{m-2}\right)$ in $K, \zeta=\theta_{m-1}$, $\eta=\theta_{m}$ and $L=k\left(y, y^{\prime}\right)$. Since $m$ was picked as small as possible, $y$ is not algebraic over $k$. Therefore, $\zeta$ and $\eta$ are algebraic ally dependent over $L$. We can conclude that there exists a $\xi$ satisfying the conclusions of the lemma. While $\xi$ is algebraic over $k\left(y, y^{\prime}\right)$, it is not algebraic over $k$, for otherwise $\zeta=\theta_{m-1}$ and $\eta=\theta_{m}$ would be algebraically dependent over $F\left(\theta_{1}, \cdots, \theta_{m-2}\right)$, contradicting the way they were chosen. Therefore, $y$ is algebraic over $F\left(\theta_{1}, \cdots, \theta_{m-2}, \xi\right)$ and so $F\left(y, y^{\prime}\right)$ would lie in a generalized elementary extension of $F$ of transcendence degree $\leqq m-1$.

Induction Step. Assume that the theorem is true for differential fields $E^{*}, F^{*}$ such that the transcendence degree of $E^{*}$ over $F^{*}$ is less than $n$. Again let $K$ be a generalized elementary extension of $F$, containing $E$, whose transcendence degree $m$ over $F$ is minimal with respect to all such extensions. Assume $m>n$ and choose a transcendence basis $\theta_{1}, \cdots, \theta_{m}$ of $K$ over $F$ such that each $\theta_{i}$ is a good elementary integral or a good exponential of an elementary integral with respect to an algebraic extension of $F\left(\theta_{1}, \cdots, \theta_{i-1}\right)$.

I will first show that for each $j$, with $0 \leqq j \leqq n-1, \theta_{m-j}$ is algebraic over $F\left(\theta_{1}, \cdots, \theta_{m-j-i}, y, y^{\prime}, \cdots, y^{(j)}\right)$ and that this last field has transcendence degree $m$ over $F$. This is a standard replacement argument with the above induction hypothesis in a supporting role. For $j=0$, we know $y$ is not algebraic over $F\left(\theta_{1}, \cdots, \theta_{m-1}\right)$, for otherwise $F\left(y, y^{\prime}, \cdots, y^{(n)}\right)$ would lie in a generalized elementary extension of $F$ of transcendence degree $m-1$. Since $y$ is algebraic cover $F\left(\theta_{1}, \cdots, \theta_{m}\right)$, we get $\theta_{m}$ algebraic over $F\left(\theta_{1}, \cdots, \theta_{m-1}, y\right)$ which then must have transcendence degree $m$ over $F$. Now assume $\theta_{m-k}$ is algebraic over $F\left(\theta_{1}, \cdots, \theta_{m-k-1}, y, y^{\prime}, \cdots, y^{(k)}\right)$ for $k<j$ and that this latter field has transcendence degree $m$ over $F$. $F\left(\theta_{1}, \cdots, \theta_{m}\right)$ is therefore algebraic over $F\left(\theta_{1}, \cdots, \theta_{m-j}, y, y^{\prime}, \cdots, y^{(j-1)}\right)$ and therefore $y^{(j)}$ is algebraic over this latter field. If $y^{(j)}$ were algebraic over $F\left(\theta_{1}, \cdots, \theta_{m-j-1}, y, y^{\prime}, \cdots, y^{(j-1)}\right)$, then letting $F^{*}$ be the algebraic closure of $F\left(\theta_{1}, \cdots, \theta_{m-j-1}\right)$ in $K$ and $E^{*}=F^{*}\left(y, y^{\prime}, \cdots, y^{(j)}\right)$ we would have a field $E^{*}$ of transcendence degree $j<n$ over $F^{*}$ which lies in $K$, a generalized elementary extension of $F^{*}$, so $F^{*}\left(y, y^{\prime}, \cdots, y^{(n)}\right)$ would lie in a generalized elementary extension $K^{*}$ of $F^{*}$ of transcendence degree $j$ over $F^{*}$. $K^{*}$ would then be a generalized elementary extension of $F$ of transcendence degree $m-1$ over $F$ which contains $F\left(y, y^{\prime}, \cdots, y^{(n)}\right)$, a contradiction. So $y^{(j)}$ must not be algebraic over $F\left(\theta_{1}, \cdots, \theta_{m-j-1}, y, y^{\prime}, \cdots, y^{(j-1)}\right)$ and therefore $\theta_{m-j}$ is algebraic over $F\left(\theta_{1}, \cdots, \theta_{m-j-1}, y, \cdots, y^{(j)}\right)$ and this latter field still has transcendence degree $m$ over $F$. 
In particular, we can conclude that $\theta_{m-n+1}$ is algebraic over $F\left(\theta_{1}, \cdots, \theta_{m-n}, y, y^{\prime}, \cdots, y^{(n-1)}\right)$ and so $\theta_{m}$ and $\theta_{m-n+1}$ are algebraically dependent over $F\left(\theta_{1}, \cdots, \theta_{m-n-1}, y, y^{\prime}, \cdots, y^{n-1)}\right)$. We will now apply the lemma. Let $k$ be the algebraic closure of $F\left(\theta_{1}, \cdots, \theta_{m-n-1}\right)$ in $K$, $L$ be the algebraic closure of $k\left(y, \cdots, y^{(n-1)}\right.$ in $K, \zeta=\theta_{m-n}$, and $\eta=\theta_{m-n+1}$. By the lemma, there is a $\xi$ in $K$ such that $\xi$ is an elementary integral or an exponential of an elementary integral with respect to $\bar{k}$ and $\theta_{m-n+1}, \theta_{m-n}$ are algebraically dependent over $k(\xi)$. $\xi$ is algebraic over $F\left(\theta_{1}, \cdots, \theta_{m-n-1}, y, \cdots, y^{(n-1)}\right)$ but it is not algebraic over $F\left(\theta_{1}, \cdots, \theta_{m-n-1}, y, \cdots, y^{(n-2)}\right)$. If it were, then $F\left(\theta_{1}, \cdots, \theta_{m-n-1}\right.$, $\left.\xi, \theta_{m-n}, \theta_{m-n+1}, y, \cdots, y^{(n-2)}\right)$ would have transcendence degree $m-1$ over $F$, contradicting the fact proven in the previous paragraph. Thus $y^{(n-1)}$ is algebraic over $F\left(\theta_{1}, \cdots, \theta_{m-n-1}, \xi, y, \cdots, y^{(n-2)}\right)$. If we now let $E^{*}$ be the algebraic closure of this latter field in $K$ and $F^{*}$ be the algebraic closure of $F\left(\theta_{1}, \cdots, \theta_{m-n-1}, \xi\right)$ in $K$, we see $E^{*}$ is a differential field of transcendence degree $n-1$ over $F^{*}$ which lies in a generalized elementary extension of $F^{*}$. Therefore $E^{*}$ lies in a generalized elementary extension of $F^{*}$ of transcendence degree $n-1$ over $F^{*}$. We can conclude that $E=F\left(y, \cdots, y^{(n)}\right)$ lies in a generalized elementary extension of $F$ of transcendence degree $m-n+n-1=m-1$, contradicting our choice of $m$.

The proofs of the final two assertions of this theorem are the same as the one above, keeping in mind the final sentence of the lemma. These last two assertions were first noticed by Koenigsberger [1], who outlined an analytic proof for the cases $n=1$ and 2.

In the next two corollaries, $C(x)$ will be the field of rational functions over the complex numbers whose derivation is given by $x^{\prime}=1$ and $c^{\prime}=0$ for all $c$ in $C$.

CoRollaRY 1. If a first order differential equation over $C(x)$ has a solution which is elementary but not algebraic over $C(x)$, then the equation has either a one parameter family of solutions of the type

$$
y=G\left(x, \varphi_{0}(x)+a_{1} \log \varphi_{1}(x)+\cdots+a_{r} \log \varphi_{r}(x)+c\right)
$$

with $c$ an arbitrary constant, the $a_{i}$ 's constants and $G$ and the $\varphi_{i}$ 's algebraic functions or the equation has a one parameter family of solutions

$$
y=G\left(x, \exp \left(\varphi_{0}(x)+a_{1} \log \varphi_{1}(x)+\cdots+a_{r} \log \varphi_{r}(x)+c\right)\right)
$$

of similar descriptions

Proof. This theorem was first proven by Mordukhai-Boltovski, 
[2] and [4, p. 86], using analytic techniques. Let $y$ be an elementary but nonalgebraic solution of the equation $F\left(x, y, y^{\prime}\right)=0$. By the theorem, we know that $y$ lies in a generalized elementary extension of $C(x)$, of transcendence degree one over $C(x)$. This means that $y$ can be considered as an algebraic function $G(x, \theta)$ where $\theta$ is an elementary integral or exponential of an elementary integral with respect to some algebraic extension $K$ of $C(x)$. If $\theta$ is an elementary integral with respect to $K$ and $c$ is any element of $C$, I claim that $G(x, \theta+c)$ is a solution of the same differential equation as $y$. This can be stated algebraically. The map which takes $\theta$ to $\theta+c$ induces differential automorphism (i.e. a field theoretic isomorphism which preserves the differential structure) of $K(\theta)$. This map can be extended to a (field theoretic) isomorphism is $K(\theta, y)$ into $\overline{K(\theta)}$, the algebraic closure of $K(\theta)$. It is known [5], that the differential structure of an algebraic extension of a differential field of characteristic zero, is uniquely determined, so the isomorphism of $K(\theta, y)$ into $\overline{K(\theta)}$ is a differential isomorphism. Therefore the image of $y$ satisfies the same differential relationships over $K$ as $y$ does. The image of $y=G(x, \theta)$ is just $G(x, \theta+c)$ so this proves the claim. If $\theta$ is an exponential of an elementary integral, then for any nonzero $d$ in $C$ the map which takes $\theta$ to $d \theta$ induces a differential automorphism of $K(\theta)$. The same kind of reasoning tells us that $G(x, d \theta)$ satisfies the same equation as $G(x, \theta)$. We therefore have the conclusion of the corollary.

CoROLlaRY 2. Let $f(y)$ be a rational function in the indeterminant $y$ with coefficients in the complex numbers. If $y^{\prime}=f(y)$ has a nonconstant solution, elementary over $C(x)$, then $1 / f(y)$ is either of the form

$$
\frac{d(v(y))}{d y} \text { or } \quad c \frac{d(u(y)) d y}{u(y)}
$$

where $v(y), u(y)$ are rational functions of $y$ with coefficients in the complexes and $c$ is a nonzero complex number. Conversely, if $f(y) \neq 0$ and $1 / f(y)$ is of one of the two above forms, then $y^{\prime}=f(y)$ has a nonconstant solution, elementary over $C(x)$.

Proof. We prove the converse first. If $1 / f(y)$ is of the first form, we let $y$ be defined by the equation $v(y)=x$. If $y$ is of the second form, we let $y$ be defined by the equation $u(y)=\exp (x / c)$. In both cases, $y$ will be a nonconstant solution elementary of $C(x)$.

Now let $y$ be a nonconstant element of some elementary extension of $C(x)$ such that $y^{\prime}=f(y)$. We then can conclude that the simple 
transcendental extension $C(y)$, with the induced derivation ', is a differential field. Expanding $1 / f(y)$ in partial factions with respect to $y$ we get

$$
\begin{aligned}
1 & =\frac{y^{\prime}}{f(y)}=\frac{d(v(y))}{d y} y^{\prime}+\Sigma c_{i} \frac{d\left(u_{i}(y)\right) / d y}{u_{i}(y)} y^{\prime} \\
& =v(y)^{\prime}+\Sigma c_{i} \frac{\left(u_{i}(y)\right)^{\prime}}{u_{i}(y)}
\end{aligned}
$$

where $u_{i}(y), v(y)$ are in $C(y)$ and the $c_{i}$ 's are in $C$. As before, we can assume that the $c_{i}$ 's are linearly independent over $Q$. Since $y$ lies in an elementary extension of $C(x)$ we are reduced to one of the following three cases:

(1) $y$ is algebraic over $C(x)$. In this case we have a relation of the form $v^{\prime}+\Sigma c_{i}\left(u_{i}^{\prime} / u_{i}\right)=1$ in an algebraic extension of $C(x)$. Applying the proposition, with $L=C$ and $\zeta=x$, we see that the $u_{i}$ are in $C$, so $u_{i}^{\prime}=0$. Therefore

$$
\frac{y^{\prime}}{f(y)}=v^{\prime}=\frac{d(v(y))}{d y} y^{\prime} \text { or } \frac{1}{f(y)}=\frac{d(v(y))}{d y} .
$$

(2) $y$ is algebraic over $L(\theta)$ where $L$ is an algebraic extension of $C(x)$ and $\theta$ is an elementary integral with respect to $L$. Since $\theta^{\prime} \in L$, we can apply the proposition to $\zeta=\theta$ and get that each $u_{i}$ is algebraic over $C(x)$. If $d\left(u_{i}(y)\right) / d y \neq 0$ for some $i$, then $y$ would be algebraic over $C(x)$ and we would be reduced to the provious case. So we can assume $d\left(u_{i}(y)\right) / d y=0$ for all $i$ and therefore $\left(u_{i}(y)\right)^{\prime}=0$. As before we can then conclude that $1 / f(y)=d(v(y)) / d y$.

(3) $y$ is algebraic over $L(\theta)$ where $L$ is an algebraic extension of $C(x)$ and $\theta$ is a good exponential of an elementary integral with respect to $L$. First notice that if we write

$$
\frac{\theta^{\prime}}{\theta}=\Sigma d_{i} \frac{s_{i}^{\prime}}{s_{i}}+t^{\prime}
$$

with $s_{i}$ and $t$ in $L$ and $d_{i}$ in $C$, then since $\theta$ and $x$ are algebraically dependent over $C(y)$ and $x^{\prime}=1 \in C(y)$, we can apply the proposition and get that each $s_{i}$ must be algebraic over $C(y)$. Thus each $s_{i}$ is algebraic over both $C(x)$ and $C(y)$ and so must be in $C$ if we are not to be reduced to the case where $y$ is algebraic over $C(x)$. So $s_{i}^{\prime}=0$ for each $s_{i}$, and $\theta^{\prime} / \theta=t^{\prime}$. Our next step is to notice that we have a relation of the form

$$
\Sigma c_{i} \frac{u_{i}^{\prime}}{u_{i}}+v^{\prime}=1
$$

in an algebraic extension of $L(\theta)$. Since $\theta^{\prime} / \theta$ is in $L$, we can apply 
the proposition and conclude that there are integers $\nu_{0} \neq 0, \nu_{1}, \cdots, \nu_{k}$ such that each $u_{i}^{\nu} \theta^{\nu_{i}}$ and $v$ is algebraic over $C(x)$. Again if $d(v(y)) / d y \neq 0$ then $y$ would be algebraic over $C(x)$ and we would be reduced to the first case. So we can assume $v^{\prime}=(d(v(y)) / d y) y^{\prime}=0$. If we let $u_{i}^{\nu} \theta^{\nu i}=A_{i}$ then

$$
\frac{u_{2}^{\prime}}{u_{i}}=\frac{1}{\nu_{0}}\left(\frac{A_{i}^{\prime}}{A_{\imath}}-\nu_{i} \frac{\theta^{\prime}}{\theta}\right)=\frac{1}{\nu_{0}}\left(\frac{A_{i}^{\prime}}{A_{i}}-\nu_{i} t^{\prime}\right)
$$

so

$$
1=\Sigma c_{i} \frac{u_{i}^{\prime}}{u_{i}}+v^{\prime}=\frac{1}{\nu_{0}} \Sigma c_{i} \frac{A_{i}^{\prime}}{A_{i}}-\frac{1}{\nu_{0}}\left(\Sigma c_{i} \nu_{\imath}\right) t^{\prime} .
$$

Each $A_{i}$ and $t$ is algebraic over $C(x)$ and $x^{\prime} \in C$ so applying the lemma for the final time we can conclude that each $A_{i}$ is in $C$. Thus, for each $u_{i}$,

$$
\frac{u_{i}^{\prime}}{u_{i}}=-\frac{\nu_{i}}{\nu_{0}} \frac{\theta^{\prime}}{\theta}
$$

If all the $\nu_{i}$ where 0 , then each $u_{i}^{\prime}=0$, so we would have

$$
1=\Sigma c_{i} \frac{u_{i}^{\prime}}{u_{i}}+v^{\prime}=0
$$

a contradiction. We can therefore assume $\nu_{1} \neq 0$ and then $u_{i}^{\prime} / u_{i}=$ $\left(\nu_{i} / \nu_{1}\right)\left(u_{1}^{\prime} / u_{1}\right)$ so

$$
\Sigma c_{i} \frac{u_{i}^{\prime}}{u_{i}}=\left(\Sigma c_{i} \frac{\nu_{i}}{\nu_{1}}\right) \frac{u_{1}^{\prime}}{u_{1}}
$$

Finally we get

$$
\frac{y^{\prime}}{f(y)}=\Sigma c_{\imath} \frac{u_{i}^{\prime}}{u_{i}}+v^{\prime}=c \frac{u_{1}^{\prime}}{u_{1}} \text { where } \quad c=\Sigma c_{\imath} \frac{\nu_{i}}{\nu_{1}} .
$$

Thus $1 / f(y)=c\left(d\left(u_{1}(y) / d y\right) / u_{1}(y)\right.$.

We can use Corollary 2 to show certain differential equations have no elementary solutions. First notice that if we can write $1 / f(y)=d(v(y)) / d y$ for some $v(y)$ in $C(y)$, then by expanding $v(y)$ in powers of $y$ and differentiating term by term we see that $1 / f(y)$ could not have any term of the form $1 / y$, when we expand $1 / f(y)$ in powers of $y$. Similarly if $1 / f(y)=(d(u(y)) / d y) / u(y)$, when we expand $1 / f(y)$ in powers of $y$, no terms of the form $y^{i}$ for $i<-1$ can appear. In particular $y^{\prime}=y^{2} /(y+1)$ has no nonconstant elementary solutions since $(y+1) / y^{2}=\left(1 / y^{2}\right)+(1 / y)$. In general if $f(y) \in Q(y)$, we can decide if $1 / f(y)$ is of one of the two forms described in the corollary as was shown by Risch [3]. 


\section{REFERENCES}

1. L. Koenigsberger, Bemerkungen zu Liouville's Classificirung der Transcendenten, Math. Ann., 28 (1887), 482-492.

2. D. Mordukhai-Boltovski, Sur la Resolution des Equations Differentielles du Premier Order en form Finie, Rend. Cir. Mat. Palermo, 61 (1937), 49-72.

3. R. Risch, The problem of integration in finite terms, Trans. Amer. Math. Soc., 139 (1969) 167-189.

4. J. F. Ritt, Integration in Finite Terms, Columbia University Press, New York, 1948.

5. M. Rosenlicht, Liouville's theorem on functions with elementary integrals, Pacific J. of Math., 24 (1968), 153-161.

6. - On the explicit solvability of certain transcendental equations, Inst. Haute Etudes Sci. Publ. Math., 36 (1969), 15-22.

7. A. Seidenberg, Some Basic Theorems in Differential Algebra (Characteristic $p$, arbitrary), Trans. Amer. Math. Soc., 6 (1952), 174-190.

8. M. Singer, Functions Satisfying Elementary Relations, To appear.

Received April 2, 1975 and in revised form July 15, 1975.

State University of New York-Stony Brook 



\title{
PACIFIC JOURNAL OF MATHEMATICS
}

\section{EDITORS}

RichaRd ARENS (Managing Editor)

University of California

Los Angeles, California 90024

\section{R. A. Beaumont}

University of Washington

Seattle, Washington 98105
J. DUGUNDJI

Department of Mathematics University of Southern California Los Angeles, California 90007

D. Gilbarg and J. Milgram

Stanford University

Stanford, California 94305

\section{ASSOCIATE EDITORS}
E. F. BECKENBACH
B. H. NeUmanN
F. WOLF
K. YoshIDA

\section{SUPPORTING INSTITUTIONS}

\author{
UNIVERSITY OF BRITISH COLUMBIA \\ CALIFORNIA INSTITUTE OF TECHNOLOGY \\ UNIVERSITY OF CALIFORNIA \\ MONTANA STATE UNIVERSITY \\ UNIVERSITY OF NEVADA \\ NEW MEXICO STATE UNIVERSITY \\ OREGON STATE UNIVERSITY \\ UNIVERSITY OF OREGON \\ OSAKA UNIVERSITY
}

\author{
UNIVERSITY OF SOUTHERN CALIFORNIA \\ STANFORD UNIVERSITY \\ UNIVERSITY OF TOKYO \\ UNIVERSITY OF UTAH \\ WASHINGTON STATE UNIVERSITY \\ UNIVERSITY OF WASHINGTON \\ AMERICAN MATHEMATICAL SOCIETY
}

The Supporting Institutions listed above contribute to the cost of publication of this Journal, but they are not owners or publishers and have no responsibility for its content or policies.

Mathematical papers intended for publication in the Pacific Journal of Mathematics should be in typed form or offset-reproduced, (not dittoed), double spaced with large margins. Underline Greek letters in red, German in green, and script in blue. The first paragraph or two must be capable of being used separately as a synopsis of the entire paper. Items of the bibliography should not be cited there unless absolutely necessary, in which case they must be identified by author and Journal, rather than by item number. Manuscripts, in triplicate, may be sent to any one of the editors. Please classify according to the scheme of Math. Reviews, Index to Vol. 39. All other communications should be addressed to the managing editor, or Elaine Barth, University of California, Los Angeles, California, 90024.

The Pacific Journal of Mathematics expects the author's institution to pay page charges, and reserves the right to delay publication for nonpayment of charges in case of financial emergency.

100 reprints are provided free for each article, only if page charges have been substantially paid. Additional copies may be obtained at cost in multiples of 50 .

The Pacific Journal of Mathematics is issued monthly as of January 1966. Regular subscription rate: $\$ 72.00$ a year (6 Vols., 12 issues). Special rate: $\$ 36.00$ a year to individual members of supporting institutions.

Subscriptions, orders for back numbers, and changes of address should be sent to Pacific Journal of Mathematics, 103 Highland Boulevard, Berkeley, California, 94708.

PUBLISHED BY PACIFIC JOURNAL OF MATHEMATICS, A NON-PROFIT CORPORATION

Printed at Kokusai Bunken Insatsusha (International Academic Printing Co., Ltd.), 8-8, 3-chome, Takadanobaba, Shinjuku-ku, Tokyo 160, Japan.

Copyright (C) 1975 by Pacific Journal of Mathematics Manufactured and first issued in Japan 


\section{Pacific Journal of Mathematics}

\section{Vol. 59, No. $2 \quad$ June, 1975}

Aharon Atzmon, A moment problem for positive measures on the unit disc ........

Peter W. Bates and Grant Bernard Gustafson, Green's function inequalities for

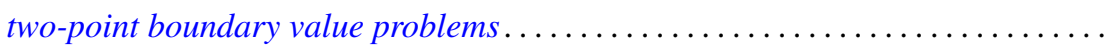

Howard Edwin Bell, Infinite subrings of infinite rings and near-rings ...........

Grahame Bennett, Victor Wayne Goodman and Charles Michael Newman, Norms of

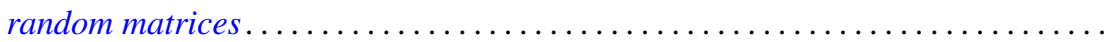

Beverly L. Brechner, Almost periodic homeomorphisms of $E^{2}$ are periodic.........

Beverly L. Brechner and R. Daniel Mauldin, Homeomorphisms of the plane ........

Jia-Arng Chao, Lusin area functions on local fields ......................

Frank Rimi DeMeyer, The Brauer group of polynomial rings ...............

M. V. Deshpande, Collectively compact sets and the ergodic theory of

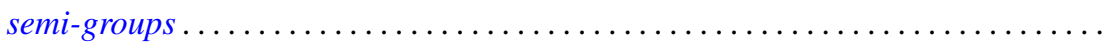

Raymond Frank Dickman and Jack Ray Porter, $\theta$-closed subsets of Hausdorff

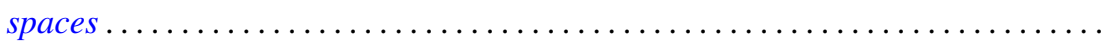

Charles P. Downey, Classification of singular integrals over a local field .......... 407

Daniel Reuven Farkas, Miscellany on Bieberbach group algebras . . . . . . . . . . . .

Peter A. Fowler, Infimum and domination principles in vector lattices . . . . . . . . . .

Barry J. Gardner, Some aspects of T-nilpotence. II: Lifting properties over

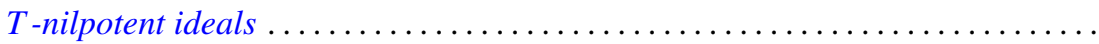

Gary Fred Gruenhage and Phillip Lee Zenor, Metrization of spaces with countable

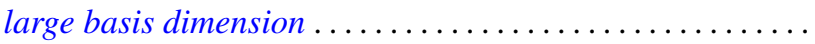

J. L. Hickman, Reducing series of ordinals...

Hugh M. Hilden, Generators for two groups related to the braid group ...

Tom (Roy Thomas Jr.) Jacob, Some matrix transformations on analytic sequence

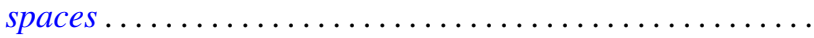

Elyahu Katz, Free products in the category of $k_{w}$-groups . .....

Tsang Hai Kuo, On conjugate Banach spaces with the Radon-Nikodým property...

Norman Eugene Liden, $K$-spaces, their antispaces and related mappings ...

Clinton M. Petty, Radon partitions in real linear spaces ........

Alan Saleski, A conditional entropy for the space of pseudo-Menger maps ....

Michael Singer, Elementary solutions of differential equations .

Eugene Spiegel and Allan Trojan, On semi-simple group algebras. I. . .

Charles Madison Stanton, Bounded analytic functions on a class of open Riemann

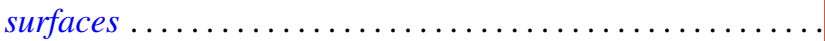

Sherman K. Stein, Transversals of Latin squares and their generalizations ....

Ivan Ernest Stux, Distribution of squarefree integers in non-linear sequences . . .

Lowell G. Sweet, On homogeneous algebras ................

Lowell G. Sweet, On doubly homogeneous algebras .......... .

Florian Vasilescu, The closed range modulus of operators ......

Arthur Anthony Yanushka, A characterization of the symplectic groups $\operatorname{PSp}(2 m, q)$

as rank 3 permutation groups... 